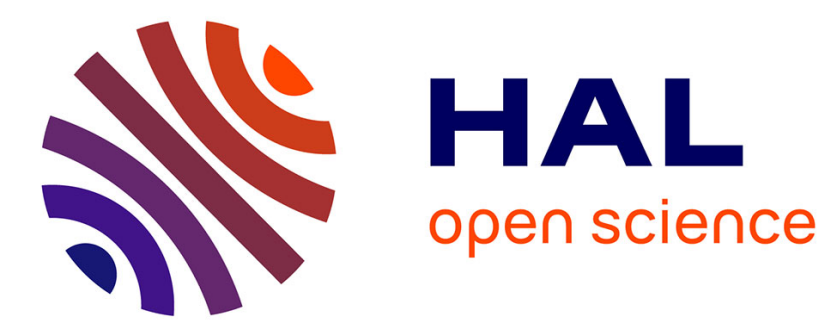

\title{
La restauration du perron nord de la terrasse des Éléphants à Angkor Thom. Rapport sur la première année de travaux (avril 1996-avril 1997)
}

Christophe Pottier

\section{- To cite this version:}

Christophe Pottier. La restauration du perron nord de la terrasse des Éléphants à Angkor Thom. Rapport sur la première année de travaux (avril 1996-avril 1997). Bulletin de l'Ecole française d'ExtrêmeOrient, 1997, 84 (1), pp.376-401. 10.3406/befeo.1997.3821 . halshs-01989543

\section{HAL Id: halshs-01989543 \\ https://shs.hal.science/halshs-01989543}

Submitted on 22 Jan 2019

HAL is a multi-disciplinary open access archive for the deposit and dissemination of scientific research documents, whether they are published or not. The documents may come from teaching and research institutions in France or abroad, or from public or private research centers.
L'archive ouverte pluridisciplinaire HAL, est destinée au dépôt et à la diffusion de documents scientifiques de niveau recherche, publiés ou non, émanant des établissements d'enseignement et de recherche français ou étrangers, des laboratoires publics ou privés.

\section{()ㅜ) $\Theta$}

Distributed under a Creative Commons Attribution - NoDerivatives| 4.0 International 
La restauration du perron nord de la terrasse des Éléphants à Angkor Thom. Rapport sur la première année de travaux (avril 1996-avril 1997)

Christophe Pottier

\section{Citer ce document / Cite this document :}

Pottier Christophe. La restauration du perron nord de la terrasse des Éléphants à Angkor Thom. Rapport sur la première année de travaux (avril 1996-avril 1997). In: Bulletin de I'Ecole française d'Extrême-Orient. Tome 84, 1997. pp. 376-401; doi : https://doi.org/10.3406/befeo.1997.3821

https://www.persee.fr/doc/befeo_0336-1519_1997_num_84_1_3821

Fichier pdf généré le 17/01/2019 


\section{La restauration du perron nord de la terrasse des Éléphants à Angkor Thom. Rapport sur la première année de travaux (avril 1996 - avril 1997)}

Dans la continuité de la restauration de la terrasse du Roi lépreux, dont l'inauguration a été célébrée le 7 mars 1996 en présence de M. Godfrain, ministre français de la Coopération, l'EFEO a entrepris en avril 1996 la restauration du perron voisin, le perron nord de la terrasse des Éléphants.

Cet édifice résulte d'une histoire complexe faite de multiples rajouts et présente de nombreux reliefs uniques à Angkor. Bien qu'ayant déjà fait l'objet de divers travaux de restauration par le passé, ce perron se trouvait dans un état peu satisfaisant, tant esthétiquement que structurellement. Les blocs épars, les lacunes et les remontages erronés que présentait ce perron déséquilibraient profondément le très bel espace formé avec la terrasse du Roi lépreux. La mise valeur de cette zone des terrasses royales nécessitait donc un traitement similaire pour le perron nord. Par ailleurs, plusieurs parties de celui-ci montraient des assemblages instables, en particulier sur sa face orientale, victime de la chute d'un arbre en 1989.

À la demande des autorités cambodgiennes, l'EFEO a élaboré un projet de restauration qui a été approuvé par la Comité International de Coordination en décembre 1995. Prévu sur une période de deux ans avec un budget global de 759200 FRF du Ministère français des Affaires étrangères, le chantier de restauration du perron nord a commencé en avril 1996 sous la responsabilité de Jacques Dumarçay et de Christophe Pottier.

Au terme de la première année, ce rapport présente l'état d'avancement des travaux. On rappellera dans un premier temps le contexte historique et les restaurations antérieures qui sont des éléments déterminants de la démarche de restauration actuelle. Le rapport présentera ensuite pour chacune des parties du perron, un état des lieux détaillé, les interventions prévues en 1995 et les travaux réalisés depuis avril 1996, leur progression et les nouvelles informations collectées sur l'histoire complexe de l'édifice et de son environnement.

\section{Rappel historique}

\section{Les terrasses royales}

Au centre du complexe urbain d'Angkor Thom, les terrasses royales, composées de la terrasse des Éléphants et de la terrasse du Roi lépreux, forment un ensemble qui borde la face orientale du Palais royal et domine la Place royale. Elles se présentent comme de hauts soubassements continus aux parements de grès intégralement ornés de reliefs, qui supportaient une succession d'édifices en bois couverts de tuiles, édifices qui sont aujourd'hui disparus. Déployé sur plus de $500 \mathrm{~m}$ de long, cet ensemble monumental se développe suivant un jeu complexe de composition basée sur une hiérarchisation d'axes de symétrie: un axe de symétrie majeur - l'axe oriental du Palais Royal, marqué par un perron principal - et deux axes de symétrie secondaires à $150 \mathrm{~m}$ de part et d'autre - la terrasse du Roi lépreux au nord et un perron presque entièrement disparu au sud, en avant du gopura oriental du Bapùon. De part et d'autre de ces deux massifs se développaient des perrons latéraux: au nord, le perron nord de la terrasse du Roi lépreux et le perron nord de la terrasse des Éléphants, et au sud, le perron sud de la terrasse des Éléphants et un perron extrême sud réduit aujourd'hui à quelques assises sculptées au sud du gopura oriental du Bapùon. La dénomination actuelle de ces édifices, 
distinguant terrasses du Roi lépreux et des éléphants, et la destruction quasi complète des extensions sud rendent ce schéma peu intelligible.

Les dégagements, les fouilles et les restaurations réalisés par la Conservation d'Angkor depuis le début du siècle ont permis de mettre en valeur les diverses parties de cet ensemble monumental. Mais alors qu'apparaissait ce plan majestueux, les travaux contredisaient l'idée d'une conception homogène en révélant les traces d'une histoire complexe de modifications. Les deux murs de reliefs sculptés de la terrasse du Roi lépreux, découverts en 1911, en sont un exemple remarquable. Les restaurations de B.P. Groslier en 1969 ont par ailleurs montré trois états successifs au perron extrême nord de la terrasse du Roi lépreux, et deux au perron sud de la terrasse des Éléphants. À la suite de ses travaux, B.P. Groslier avait proposé en 1969 une synthèse ${ }^{82}$ de l'histoire architecturale des terrasses royales que l'on résumera schématiquement ici: un premier état des terrasses est édifié en latérite dès le début du XI siècle ; endommagé par le sac Cham en 1177, ce premier état est recouvert au début du règne de Jayavarman VII sous de nouvelles terrasses parementées en grès, modifiées à plusieurs reprises durant les premières années du XIII ${ }^{e}$ siècle; de nouvelles transformations furent encore effectuées entre le XIVe et le XVI' siècle.

\section{Le perron nord de la terrasse des Éléphants}

Le perron nord de la terrasse des Éléphants est loin de faire exception. Il nous apparaît même exemplaire de cette histoire complexe puisqu'il présente aussi un enchevêtrement complexe de divers états mis au jour au fil des travaux et des sondages qui furent particulièrement nombreux pour une structure aussi réduite. Mais pour mieux se repérer dans cette superposition, il n'est pas inutile de préciser la configuration particulière du perron et la terminologie que nous employons dans ce rapport.

Les recherches menées cette année nous permettent actuellement de considérer sur la face orientale l'existence de six structures distinctes, révélatrices d'époques diverses (fig. 1):

- M0 est composé d'un mur de latérite qui délimite un état primitif du perron,

- M1 recouvre M0 dans son intégralité par un parement de grès sculpté,

- M2 masque l'extension orientale de M1 par un nouveau parement de grès sculpté,

- M3 recouvre la face est de M2 sous une nouvelle extension,

- M4 est un panneau de reliefs situé sur la plate-forme du perron, de M4.

- le stupa, dernier état, est construit sur un massif de latérite qui chevauche la face arrière

Nous devons aussi mentionner l'existence du mur du « retour nord » qui borde la face sud du passage entre le perron et la terrasse du Roi lépreux. Nous désignerons enfin sous le terme général de «face ouest » l'ensemble des structures inédites dégagées cette année dans la partie occidentale du perron.

\section{Restaurations antérieures}

Cette imbrication d'états successifs forme sans conteste l'un des deux aspects originaux du présent programme de restauration. La seconde particularité, tout aussi contraignante que la première, réside dans le nombre important de réparations, de fouilles, d'effondrements, de consolidations et de reprises ponctuelles qu'a connu cet édifice. À tel point que rares sont les parties authentiques qui n'aient jamais été touchées. On rappellera donc les éléments essentiels de l'histoire de ces travaux qui ont d'ailleurs permis de mettre au jour l'enchevêtrement de structures successives.

82. «La terrasse du Roi lépreux », Nokor Khmer, Nc1, Phnom Penh, 1969, p. 18-33. Bien que de nombreux points qui y sont avancés restent judicieux, les travaux entrepris depuis 1993 dans cette zone permettent de revoir quelques datations et explications relatives à certains remaniements. 
1908: PREMIERS CONSTATS ET DÉGAGEMENTS

Avant même la création de la Conservation d'Angkor en 1908, les traces « maladroites de réfection » antérieures furent constatées en de nombreuses parties des terrasses royales et au perron nord en particulier. En plusieurs points, principalement sur les zones hautes, le parement de grès montrait - et montre encore - la marque de remontages hâtifs et peu soignés, quelques blocs d'origine ayant été réutilisés sans ordre particulier ni retaille, complétés de blocs étrangers, laissant dans la maçonnerie des joints béants ou partiellement obturés par des fragments de grès ou de latérite. Nous ignorons actuellement la date de ces «premières restaurations ».

En 1908 et 1911, les premiers débroussaillements permettent de dégager le sommet du perron et d'y observer « une sorte de phnom circulaire sur un haut soubassement carré en grès très décoré par 6 éléphants et dans les intervalles de vigoureux bas-reliefs » (respectivement stupa et M4).

1916-1934 : RÉPARATIONS ET SONDAGES PONCTUELS

En juillet 1916, H. Marchal ouvre un chantier de dégagement et de consolidation. À cette occasion est trouvé dans le massif oriental du perron nord un panneau de reliefs présentant un cheval pentacéphale (moitié nord de la face orientale de M2), premier vestige d'un état antérieur ${ }^{83}$.

Lors des débats qui suivent la publication de la thèse de P. Stern, H. Marchal, « désireux de mettre au point ou tout au moins d'essayer de jeter un peu de lumière sur certains endroits restés énigmatiques », réalise plusieurs sondages: en 1927 et 1929 à l'angle sud de M4, en 1932 au nord est de l'escalier nord. Par ailleurs, à la suite d'effondrements et de la chute d'un arbre sur le sommet du perron, il réalise des remontages ponctuels avec consolidations: en 1930 à l'angle nord-est de M2 et en 1934 sur la moitié nord de M4. En 1934, H. Mauger fouille à son tour le massif du perron et y découvre un nouveau panneau de reliefs (face sud de l'extension orientale de M1, ré-ensevelie en 1935).

1951-1953 : SONDAGES COMPLETS ET ANASTYLOSE PARTIELLE

Dix-sept ans plus tard, en décembre 1951, H. Marchal décide de reprendre le sondage de Mauger dont seules quelques photographies témoignent. Les dégagements se veulent cette fois-ci systématiques et durent plus d'un an : ils mettent au jour l'intégralité du panneau de reliefs repéré par Mauger (M1 face sud), mais aussi son symétrique au nord (M1 face nord), un mur de latérite en arrière de ceux-ci (M0) et, vers l'est, un second panneau de reliefs présentant un cheval pentacéphale (M2 moitié sud), symétrique de celui dégagé en 1916 (M2 moitié nord). Fort de ses dégagements, H. Marchal considère alors que ce perron a connu trois états successifs mais les raisons de ces transformations restent obscures ${ }^{84}$. Quelques mois après le début des sondages, s'impose la nécessité de consolider les parties dégagées qui forment une «brèche ouverte et béante qui éventre le dallage » du perron. Ces consolidations se révèlent délicates vu l'étroite imbrication des vestiges découverts et la nécessité d'aménager une circulation et des escaliers d'accès pour permettre leur visite. Dans le prolongement de ce travail est engagée de décembre 1952 à mai 1953 la « remise en état » de l'extrémité orientale

83. Un premier escalier de latérite est ménagé en mars 1917 pour faciliter leur visite. Outre la reconstruction des parties chancelantes ou au dévers trop prononcé, les travaux portent sur la reprise des dallages supérieurs et le remontage d'un panneau de parement écroulé en août 1912 à l'angle sud du perron nord. Lors des premières pluies suivantes, une seconde reprise est nécessaire dans ce même angle et amène la découverte dans la maçonnerie d'une dalle portant les traces d'une inscription malheureusement trop effacée pour être lisible (K. 487).

84. « Modifications successives de la partie nord de la terrasse des Éléphants d'Angkor Thom », BEFEO XLVII, Paris, 1953, publié en 1955, p. 617-620. 
du perron (M3) pour pallier au manque de cohésion du parement et remédier à l'aspect « disparate [de reliefs à l'évidence] surajoutés ». Le parement des panneaux principaux est donc en partie démonté - parfois jusqu'aux assises basses (faces sud et nord) mais sans toucher les assises de fondation - puis remonté, cramponné et rejointoyé au mortier. " $\mathrm{Ce}$ travail fut assez long et suscita de nombreux tâtonnements, car [le parement avait été] fortement tripatouillé. Il était donc difficile de raccorder avec les pierres appartenant au basrelief primitif. D'autre part, un certain nombre de blocs sculptés retrouvés ça et là étaient susceptibles de venir remplacer ceux qui manquaient, d'où des essais de remise en place, parfois heureux, mais auxquels il fallait quelquefois renoncer ». À l'occasion de ces démontages, $\mathrm{H}$. Marchal note aussi la présence de nombreux blocs en réemploi, tant dans le parement que dans le massif interne composé d'un blocage grossier de latérite et de grès.

Enfin, en 1954, J. Boisselier constatant «le fruit davantage accusé chaque jour » que prenaient certains angles du perron (principalement M3 angle nord est et, dans une moindre mesure, M2 angle sud est et M1 angle sud ouest), pourtant consolidés un an auparavant, reprend leur consolidation. Par ailleurs, des naga sont installés sur le sommet des murs de parement (M2 et M3) et les échiffres de l'escalier nord sont recomposées pour accueillir deux lions « dont un retrouvé dans la brousse ».

1968 : PROGRAMME D'ANASTYLOSE INTÉGRALE DES TERRASSES ROYALES

Avec l'arrivée de B.-P. Groslier au poste de conservateur d'Angkor en 1960 et avec l'accélération des travaux au Bapùon, la restauration des terrasses royales est repoussée jusqu'en 1968 date à laquelle Groslier y engage une campagne d'anastylose intégrale : leur « restauration était à la fois la suite logique des travaux du Bapùon et plus particulièrement du pavillon d'entrée V Est de ce temple et une nécessité impérieuse. En fait elle aurait dû être entreprise depuis longtemps. Sous la pression des terres, le mur de la terrasse se gonfle et se disloque. L'eau qui stagne au pied accélère le tassement des fondations. Quoique demandant un gros travail, la reconstruction intégrale des terrasses s'imposait ». Elle commence alors avec deux équipes, une à chaque extrémité - au perron sud de la terrasse des Éléphants et à la terrasse du Roi lépreux - censées se rejoindre à la fin au perron central. Mais les travaux sont interrompus en 1970 alors que le chantier sud est quasiment achevé et que seule l'extrémité nord de la terrasse du Roi lépreux est terminée.

La terrasse du Roi lépreux a fait l'objet du premier chantier entrepris par l'EFEO après sa réimplantation à Angkor en 1992 et le monument restauré a été rendu au public en mars 1996. La restauration du perron nord de la terrasse des Éléphants s'inscrit alors dans la continuité de ce programme de mise en valeur et de restauration des terrasses royales amorcé il y a 30 ans.

\section{Projet de restauration et travaux réalisés}

Le projet de restauration du perron nord de la terrasse des Éléphants soumis en 1995 au Comité International de Coordination vise à assurer la stabilité des diverses parties de cet édifice et une meilleure pérennité de ses reliefs tout en mettant en valeur tant sa complexité historique que ses formes architecturales. Ce projet s'attache particulièrement à la diversité des éléments constitutifs de ce perron qui, comme on l'a vu plus haut, résulte d'une superposition d'états divers. Or ces éléments se présentent actuellement dans des conditions de conservation très variables. La restauration se devait d'offrir une réponse adéquate aux besoins structurels de chaque structure, et soucieuse de la mise en valeur de leurs caractéristiques. Elle a donc été envisagée en tant qu'ensemble d'interventions différenciées et adaptées aux pathologies structurelles de chaque partie et à leurs valeurs architecturales et historiques.

Le projet présente plusieurs thèmes qui, au niveau du chantier, restent étroitement mêlés : dégagement des parties effondrées, relevés topographiques, graphiques et photographiques, consolidations en sous-œuvre sans démontage et restaurations par anastylose. 
Le projet prévoyait le planning suivant sur deux ans :

- première année : relevé général de l'ensemble, dégagement des parties effondrées de M3, consolidations de M2, début des anastyloses de M1, du retour nord, de M4 et du stupa.

- seconde année : anastylose de M3, fin des anastyloses de M1, du retour nord, de M4 et du stupa, et traitement des abords et de la face ouest.

Les travaux de restauration entrepris cette année diffèrent sensiblement de ce planning prévisionnel pour deux raisons majeures. La première réside dans le budget alloué pour cette première année: 185185 FRF contre 388000 FRF prévus. Soit $25 \%$ du budget prévisionnel total. Celui-ci ne comprenait que la main d'œuvre et les matériaux, les équipements ayant été précédemment acquis pour la terrasse du Roi lépreux. Cette baisse de budget s'est donc répercutée directement tant sur le nombre d'ouvriers que sur l'ampleur des travaux. La seconde raison concerne les configurations nouvelles et particulières apparues en cours de chantier : les sondages préliminaires aux travaux ont permis de constater une bonne stabilité de certaines structures (M2 et partiellement M1) et donc de revoir en partie à la baisse certaines interventions prévues. Mais ces sondages ont d'autre part révélé d'importantes structures inédites (en particulier sur la face ouest) et de nouveaux besoins de consolidation. Ces nouvelles données, s'équilibrant en partie sans remettre en cause l'enveloppe du projet, nous ont amené à modifier certaines opérations et leur planning afin de rester fidèles au principe d'adaptation que le projet s'était donné.

Les travaux réalisés depuis avril 1996 se sont donc concentrés sur les éléments suivants :

- Relevé de l'ensemble.

- M3 : dégagement des parties effondrées et anastylose.

- Retour nord : anastylose.

- Face ouest : sondages, dégagements des structures et consolidation en sous-œuvre de la moitié nord.

- M4 et stupa : début de l'anastylose.

- M1 et M2: sondages.

Nous considérerons ci-après, pour chacune de ces tâches, l'état avant travaux, les travaux réalisés, les informations nouvelles mises au jour, et les travaux prévus pour la seconde année.

\section{Préparation du chantier}

L'équipe cambodgienne du chantier du perron nord de la terrasse des Éléphants est constituée des meilleurs éléments de l'équipe qui travaillait à la terrasse du Roi lépreux depuis 1993. Elle est désormais réduite à 67 personnes : 1 assistant, 1 chef d'équipe, 4 dessinateurs, 4 tailleurs de pierre, 52 ouvriers, 2 chauffeurs et 3 gardiens. L'organisation du travail suit les principes développés à la terrasse du Roi lépreux et au Bapùon ; le chantier est en activité de $7 \mathrm{~h}$ à $14 \mathrm{~h}$, sauf cas exceptionnels. L'ensemble du périmètre du chantier a été clairement délimité et son accès interdit au public pour raison de sécurité.

Le chantier bénéficie par ailleurs des facilités et des structures offertes par le centre de l'EFEO à Siemreap.

Les travaux réalisés font l'objet de rapports mensuels remis aux autorités cambodgiennes (APSARA et Conservation d'Angkor) et à l'UNESCO.

\section{Relevé architectural}

Avant le début des travaux, l'équipe de géomètres et de dessinateurs de l'EFEO a complété le relevé topographique au $1 / 100^{\circ}$ fait en avril 1995 pour l'étude préliminaire, et a étendu la trame orthogonale de référence utilisée à la terrasse du Roi lépreux à l'ensemble du perron. À partir de ces repères et après éradication de la petite végétation et nettoyage du 
monument, la couverture graphique intégrale des structures a été réalisée au $1 / 20^{\circ}$. Ces panneaux ont ensuite été assemblés au $1 / 50^{\circ}$ sous forme d'élévations et de coupes générales et ont servi de base au relevé architectural intégral du monument. Le plan d'ensemble au 1/20 du perron a été achevé en mai 1996.

$\mathrm{Au}$ fil des travaux, tous les éléments nouveaux ont été enregistrés graphiquement et, au total, 84 documents graphiques ont été dressés (plans, élévations, coupes, stratigraphies...). À ceux-ci s'ajoutent 396 relevés grandeur nature des graffiti et des traces de réemploi repérés sur les blocs constituant M3.

Parallèlement, nous avons poussé nos recherches documentaires sur l'édifice, son histoire et ses restaurations antérieures. Nous avons ainsi collecté divers articles, quelques plans fragmentaires, les rapports de travaux et toutes les photographies du fond de l'EFEO à Paris.

\section{Murdu troisième état (M3)}

ÉTAT AVANT TRAVAUX

Le troisième état, extension orientale du perron, est sans conteste l'élément qui se présentait en 1996 dans les plus mauvaises conditions ( $\mathrm{Ph}$. 1). Structurellement, les restaurations précédentes s'étaient attachées à rendre une cohésion à l'appareillage allègrement «tripatouillé » avant le XXe siècle (le mot est de $\mathrm{H}$. Marchal, rappelons-le). Le parement de grès, orné de reliefs (lions et garuda en atlantes, éléphant tricéphale, registre supérieur de danseuses et de divinités assises), est particulièrement fin et certains blocs ne présentent que quelques centimètres d'épaisseur. Le parement avait donc été, en 1953, en grande partie déposé et remonté avec des ancrages métalliques puis rejointoyé. Mais, outre la faiblesse des blocs et leur mauvaise condition de préservation, des tassements différentiels se produisaient très nettement aux angles et, ajoutés aux problèmes du parement, ils entraînaient le décollement de celui-ci. Ce phénomène n'a pas été l'objet de consolidations suffisantes et semble avoir continué malgré les restaurations réalisées, comme le laissent penser les nombreux joints mortoyés en 1952 qui s'étaient rouverts en 1996.

Par ailleurs, malgré les efforts successifs de $\mathrm{H}$. Marchal et de J. Boisselier pour retrouver les blocs d'origine, les assises supérieures demeuraient encore très lacunaires et comprenaient plusieurs blocs douteux et de nombreux blocs étrangers qui n'y avaient été assemblés que pour reconstituer un volume sans grand soucis de la continuité des reliefs. Notons par exemple que certains blocs sculptés de la terrasse du Roi lépreux y ont été trouvé cette année. Quant à la face septentrionale, elle se réduisait à quatre assises d'origine et une tête de lion qui chevauchait un corps de garuda.

Pour comble de malchance un grand arbre, un dipterocarpus alatus, situé à environ $5 \mathrm{~m}$ de l'angle nord-est s'est abattu en 1989 sur plus de la moitié de la face orientale, pulvérisant la majeure partie de l'angle nord-est et la moitié nord du panneau central avec les têtes de son éléphant tricéphale. Les nombreux crampons métalliques insérés dans les blocs en 1953 ont répercuté le choc et entraîné de nombreuses fêlures. Un total de 487 blocs et fragments de blocs ont été retirés des éboulis (dont trente blocs divers - têtes d'éléphants, trompes ramenés en 1993 par la Conservation d'Angkor dans son dépôt). La situation d'instabilité du sommet de la moitié nord était telle que les autorités cambodgiennes nous avaient demandé en 1995 d'en fermer l'accès au public.

En 1996, on constatait donc pour cette seule partie plusieurs problèmes importants: tassements des angles, effondrement et bris de blocs, lacunes des parties supérieures et quantité de blocs incongrus. Considérant que cet édifice avait déjà été grandement démonté en 1953, et donc que son authenticité n'était plus que relative, une anastylose était projetée. 


\section{TRAVAUX RÉALISÉS}

DÉMONTAGE

Les blocs effondrés furent tout d'abord enlevés après relevés détaillés de localisation et numérotation. Le dégagement de ces parties éboulées a été entrepris en mars et, au fur et à mesure de l'enlèvement, les blocs inférieurs étaient numérotés et relevés. Quatre couches ont ainsi été repérées, couvrant 457 blocs et fragments qui furent entreposés sur le champ de dépose oriental.

Après relevé graphique de l'appareillage au $1 / 20^{\circ}$, couverture photographique et numérotation de tous les blocs - y compris les blocs douteux et étrangers, ce qui constituera une documentation précise sur les restaurations antérieures - l'édifice à été démonté en mai et en juin 1996. Ce démontage s'est révélé délicat et long car les blocs avaient été cramponnés et scellés au mortier de ciment en 1953. Par contre, le massif n'était constitué que d'un empilage grossier de blocs de latérite et de terre.

\section{CONFORTATIONS}

Au niveau des assises de fondations, un réseau important de dépôts de fondation a été mis au jour. Une fois tous ces dépôts repérés, relevés et dégagés, la zone a pu être préparée pour la réalisation de la nouvelle dalle. Avec les pluies de mousson arrivées entre-temps - et les inondations du chantier malgré un pompage quotidien - la nouvelle fondation n'a pu être achevée qu'à l'occasion des premières accalmies de septembre. À la différence des confortations réalisées à la terrasse du Roi lépreux - qui comprenaient pour chaque mur une dalle et un mur rideau en béton armé destiné à reprendre les poussées du remblai interne haut de 6 mètres - la solution utilisée pour le troisième état du perron nord de la terrasse des Éléphants ne comprend qu'une dalle de béton armé sur laquelle est élevé un mur maçonné de latérite en arrière du parement de grès afin de maintenir celui-ci et de contenir le faible remblai interne peu élevé.

\section{REMONTAGE}

Au début d'octobre, sur la dalle de béton armé préalablement couverte d'une étanchéité, le calage des premières assises a été engagé et en mars, tous les blocs dont l'emplacement était sûr étaient remontés (photo 3). Au fur et à mesure du remontage du parement était réalisé le mur interne en blocs de latérite maçonnés au mortier léger de ciment. Ce mur a été dressé derrière chaque panneau de parement, sous les escaliers (les deux escaliers d'origine à l'est, et l'escalier neuf à l'ouest qui permet de descendre depuis le sommet de M3 jusqu'au pied des reliefs de M2), et derrière le parement de blocs de latérite appareillés à joints vifs de la face ouest. Par ailleurs étaient réalisés le drain périphérique à la base de la dalle et les descentes à l'intérieur du massif.

Le remontage du parement de grès sculptés (de 3,30 m au maximum conservé), bien que concernant une structure d'échelle réduite, a posé des problèmes d'un ordre particulier, liés à la qualité médiocre de l'appareillage d'origine et aux diverses restaurations antérieures. D'une part, les blocs sont particulièrement fragiles (altération des reliefs, faible épaisseur, nombreuses cassures...) et montrent que l'appareillage d'origine ne s'était que relativement préoccupé d'obtenir des joints parfaitement fermés, en particulier dans les assises basses. Or la cohérence de celles-ci étant essentielle lors du remontage, il a donc été continuellement nécessaire d'évaluer et de distinguer, principalement à partir des relevés précis avant démontage, les joints liés à des désordres de ceux dus à un défaut d'appareillage d'origine. D' autre part, les nombreuses interventions antérieures avec leurs divers « tripatouillages» s'étaient attachées à reconstituer les reliefs, en ne tenant que relativement compte de la stéréotomie particulière de chaque bloc, quitte parfois à retailler une face ou à laisser un joint de mortier. Les reliefs étant assez répétitifs, le résultat d'ensemble apparaissait cohérent en 
façade. Mais la repose de ces blocs a souvent montré que leur position était erronée. Nous avons donc, quasiment dès la pose des premières assises de reliefs, vérifié la position d'origine de chaque bloc et engagé des recherches pour identifier leur localisation exacte et retrouver les blocs manquants.

RECHERCHE DES BLOCS MANQUANTS

Focalisée dans un premier temps sur le perron nord, la recherche des blocs manquants s'est étendue sur une zone plus vaste, allant du nord de la terrasse du Roi lépreux au perron central de la terrasse des Éléphants. De même, pour les parties pulvérisées par la chute de l'arbre en 1989, la recherche de recollement des fragments a été complétée par une vérification de leur place d'origine, souvent différente de l'emplacement avant l'effondrement suggéré par quelques photographies d'archives.

Ces recherches, qui continuent encore pour le sommet, ont été particulièrement fructueuses pour les panneaux nord où, outre deux garuda d'angle qui avaient été intervertis et ont depuis été reconstruits - et complétés - à leur position d'origine, une proportion importante des reliefs a été retrouvée et on peut désormais envisager la reconstruction de cette zone jusqu'à une hauteur proche de son niveau originel. Pour les panneaux symétriques au sud, plus complets, des insertions approximatives réalisées en 1953 et 1954 ont été corrigées et les lacunes comblées par des blocs neufs taillés sur mesure. Dans les panneaux orientaux, où plusieurs blocs de la terrasse du Roi lépreux avaient été réutilisés - l'un d'eux retrouvera prochainement sa place d'origine récemment identifiée - le travail a été des plus complexes puisque les reliefs recherchés concernent des danseuses et des têtes d'éléphants, ce dernier sujet étant particulièrement commun dans les environs... La recherche s'est donc étendue à plusieurs groupes de blocs : blocs effondrés en morceaux, blocs ramenés à la Conservation d'Angkor, blocs provenant du dégagement de la terrasse supérieure du perron nord et de l'ensemble de la terrasse des Éléphants, blocs réutilisés lors des « restaurations » d'autres parties du perron nord, blocs mis au jour lors des sondages de la face occidentale et enfin blocs rangés aux environs de la terrasse du Roi lépreux et du perron central de la terrasse des Éléphants. Nous nous sommes cependant abstenus - pour l'instant - d'étendre nos recherches aux autres parties restaurées de la terrasse des Éléphants, bien que les diverses restaurations qui y ont été réalisées s'étaient toutes heurtées à d'importantes difficultés lors du remontage des têtes d'éléphants. Le travail de recherche des blocs de M3 dépendait étroitement d'autres parties de l'édifice, et donc de leurs restaurations. Ces recherches ont été entreprises en liaison avec les travaux de M4 et de la base du stupa, constituée d'un amas de blocs en réemploi. On peut d'ores et déjà reconstituer le sommet des têtes d'origine de l'éléphant tricéphale monumental qui orne la partie médiane de la face orientale. Ces têtes se révèlent d'ailleurs être complètement différentes de celles reconstruites en 1953 - ces dernières proviennent en fait en partie de M4 et y seront remontées. Notons enfin que, comme à la terrasse du Roi lépreux, la restauration restera « évolutive » dans le sens où il sera toujours possible de replacer ultérieurement un bloc d'origine si sa localisation venait à être trouvée.

\section{Éléments mis au jour lors des travaux}

NOUVELLE DATATION DU MUR DU TROISIÈME ÉTAT

Le démontage du parement a donné l'occasion de mettre au jour de très nombreux blocs en réemploi dont le style est voisin de celui du Bayon: pilastres avec reliefs de feuillages ou d'apsara, colonnettes octogonales incluses dans des bloc de piédroits, rampant et motif terminal de fronton polylobé, éléments de piliers appareillés avec moulures d'angles... En fait, il semble que le troisième état n'ait été composé que de blocs de réemploi. Sur quelques blocs, nous avons pu identifier avec certitude des images bouddhiques sous arcature trilobée, du même type que ceux qui ornent certains piliers du Bayon. Or ces images portent toutes des traces évidentes de bûchage. On sait par ailleurs que l'effacement de l'iconographie 
bouddhique de - presque - tous les temples à Angkor date d'une réaction brahmanique lors de la seconde moitié du XIII e siècle. Les blocs retrouvés dans M3 ont donc été réemployés nettement après cette période. Il est désormais clair que cet état ne saurait être attribué au règne de Jayavarman VII, et que nous devons repousser sa construction d'au moins une cinquantaine d'année ${ }^{85}$. Afin de documenter au mieux les observations uniques qu'a permis cette anastylose, tous les reliefs en réemploi et les graffiti notés sur les blocs ont été relevés et photographiés.

\section{DÉPÔTS DE FONDATION : EMBRYONS ET TORTUES}

La découverte majeure - ou au moins la plus spectaculaire - faite à l'occasion du démontage réside dans le dégagement d'un réseau remarquable de dépôts de fondation constitué d'objets inédits (cf. fig. 2 et photos. p. 402-407).

Au niveau des dernières assises, correspo-ndant à la surface de la fosse de fondation originelle, onze dépôts ont été mis au jour en divers emplacements :

- deux pots de bronze.

- trois « embryons », chacun contenant à l'intérieur d'un conglomérat ovoïde couvert d'une feuille de bronze des matériaux exceptionnels: or, bronze, gemmes, résines, fils d'or, de bronze et de soie, feuille végétale et graines.

- six tortues en plomb - deux petites (longueur $17 \mathrm{~cm}$ ) et quatre grandes (longueur de 45 à $65 \mathrm{~cm}$ ) - toutes alignées nord-sud sous le front oriental, les petites la tête au sud, les grandes au nord-est.

Notons cependant que les analyses que mériteraient ces objets n'ont pu être réalisées pour des raisons budgétaires. Nous ignorons donc encore la nature exacte de certains d'entre eux : fils de soie, gemmes, résines, éléments végétaux et ... l'intérieur des tortues.

Le dégagement de ces dépôts a légèrement retardé le coulage de la nouvelle fondation de béton armé : après la mise au jour du premier dépôt, ce qui n'était que simple creusement a été organisé comme une fouille archéologique afin de prendre toutes les précautions nécessaires et de réaliser les observations spécifiques et les relevés détaillés. Un sondage complémentaire a d'ailleurs été réalisé pour reconnaître le fond de la fosse de fondation originelle.

Les conditions de la mise au jour des quatre grandes tortues de plomb, les derniers dépôts à être découverts, sont assez particulières et méritent d'être notées. En effet, la dépose de la dernière assise et le remblai fouillé jusqu'à ce niveau avaient déjà permis de mettre au jour sept dépôts. Il devenait improbable d'en trouver d'autres. Il paraissait cependant nécessaire de s'en assurer avant de reconstruire l'édifice, car ceux déjà découverts montraient un réseau complexe dense à la configuration unique dans un site angkorien. Mais fouiller plus profondément le remblai impliquait de retarder encore le chantier pour d'hypothétiques découvertes (une vérification «manuelle » correspondait à dégager plus de $40 \mathrm{~m}^{3}$ ) et surtout de fragiliser la base des structures voisines de M2. Nous avons donc contacté J. P. Billault qui dirige l'équipe de déminage de la CIDEV-COFRAS et avons évalué les possibilités offertes par les détecteurs de mines. Nous avons alors préalablement fait des essais avec les dépôts déjà découverts afin de vérifier que les détecteurs étaient sensibles à des matériaux non ferreux, et ce jusqu'à quelle profondeur. Ces essais étant probants pour le bronze, le plomb et dans une moindre mesure pour l'or, une équipe de démineurs est alors venue sur le site « dévitaliser » la zone suspecte. Vingt minutes à peine furent nécessaires pour localiser les quatre tortues (et une bonne journée pour les dégager et les sortir après réalisation de relevés précis). La zone put donc après cette vérification être considérée comme vierge afin d'entreprendre les confortations nécessaires au remontage de la structure. L'implication de CIDEV-COFRAS pour le déminage des temples est déjà ancienne et bien connue. Depuis 1995, sa collaboration étroite avec la Conservation d'Angkor s'est étendue aux travaux de recherches menés à

85. Il semble que la datation de M3 ne doive cependant pas être repoussée jusqu'au XVIesiècle (cf. infra M4. p. 391). 
Angkor - et à ceux de l'EFEO en particulier. Mais l'utilisation de détecteurs dans un but proprement archéologique est assez originale. Elle a apporté, dans le contexte singulier des travaux de restauration du perron nord de la terrasse des Éléphants, un gain de temps appréciable. Mais elle y a surtout permis des dégagements ponctuels et précis, évitant ainsi de créer des risques d'affouillement pour les structures voisines. Notons enfin qu'elle n'est venue qu'en appoint, pour ôter un doute, et qu'elle ne saurait être utilisée en dehors de conditions exceptionnelles sous peine d'évoquer une chasse aux trésors hors de propos. Nous avons d'ailleurs par curiosité fait des essais dans d'autres zones et les détecteurs s'y sont révélés inopérants, trop troublés par les interférences des nombreux grains de latérite ferrugineuse qui jonchent le sol d'Angkor Thom.

Les dépôts, de par leur valeur archéologique, sont aujourd'hui gardés à la Conservation d'Angkor et n'ont bien sûr pas été remis en place avant le coulage de la dalle. Mais pour satisfaire à quelques réactions apparues après la découverte de ces dépôts sacrés, qui y voyaient un caractère «blasphématoire », nous avons réinséré des dépôts de fondation avant de reconstruire l'édifice. Il eut été futile de confectionner de pâles copies, et ridicule de singer un rituel ancien dont nous ignorons presque tout. Aussi nous avons pris le parti de la contemporanéité - évidente puisqu'il s'agissait de loger ces dépôts au niveau d'une dalle de béton armé - et nous avons suivi les us actuels. Nous avons donc ménagé des cavités lors du coulage de la dalle pour y installer les nouveaux dépôts de fondation composés... de billets de banque, ce qui est certes moins spectaculaire, mais correspond aux pratiques actuelles et reste assez symbolique. Ces dépôts ont été installés lors d'une cérémonie religieuse adéquate organisée pour l'occasion avec le Vénérable de la Pagode voisine de Tep Pranam.

\section{Travaux prévus durant la seconde année}

Au terme de cette première année de travaux, l'anastylose de ce mur de troisième état est en voie d'achèvement. Les blocs d'origine démontés ont été rétablis, et seuls quelques blocs brisés par la chute de l'arbre sur le front oriental sont en cours de consolidation avant d'être à leur tour remontés. Le massif de latérite maçonné est achevé et le travail de finition du parement occidental atteint actuellement les dernières assises supérieures et le niveau du dallage de la plate-forme. Les recherches continuent pour identifier et replacer les blocs manquants, en particulier sur l'éléphant tricéphale et les frises hautes de danseuses. Bien qu'il soit possible de trouver de nouveaux blocs lors des travaux qui seront réalisés dans d'autres parties du perron nord, une équipe de tailleurs exécute actuellement le remplissage des lacunes basses avec des blocs neufs.

Soulignons enfin que ce remontage a été relativement long mais nous permet dorénavant d'être en mesure de garantir l'emplacement de chaque bloc remonté.

\section{Retour nord}

\section{État avant travaux}

Le retour nord est un long panneau de parement de grès qui s'étend vers l'ouest depuis l'escalier nord. Avec son symétrique au nord - le retour sud de la terrasse du Roi lépreux il encadre le large passage ménagé à une époque tardive entre les deux terrasses. Comme son symétrique, ce mur porte les traces d'un remontage très approximatif de ses parties supérieures, restauration réalisée avant le $\mathrm{XX}^{\mathrm{e}}$ siècle vraisemblablement à la suite d'un effondrement. Cette réparation malhabile, du même type que celles que l'on observe ailleurs en de nombreux tronçons de la terrasse des Éléphants, n'a aucunement amélioré la stabilité du mur qui continuait à se déverser sous la pression du remblai interne. En 1996, un dévers important était notable en sa partie centrale. 


\section{Travaux réalisés}

Le projet de restauration prévoyait une anastylose de ce mur et son remontage sur une dalle et un contre-mur en béton armé. En avril 1996, une équipe venait juste d'achever le même type de restauration pour le mur symétrique de la terrasse du Roi lépreux. Elle a donc été immédiatement chargée de poursuivre ce travail au retour nord de la terrasse des Éléphants.

Après un mois de démontage, la dalle était coulée en mai et le calage des premières assises commençait. L'ensemble des blocs était remonté en juin. Comme à la terrasse du Roi lépreux, les blocs provenant de la réparation tardive avaient été remontés afin de garder la trace des remaniements antérieurs, même tardifs. Après une première campagne de recherche des blocs manquants, les extrémités ont été laissées en attente : à l'est, pour se relier à la restauration de l'escalier nord, et à l'angle ouest, pour se raccorder aux panneaux occidentaux en cours de sondages et de dégagements. Après l'achèvement des sondages de la face ouest, l'angle ouest a été repris et achevé. Alors que le parement de latérite des lacunes supérieures allait être réalisé, il est apparu que notre remontage de la réparation tardive, bien que respectant scrupuleusement les défauts des assemblages originels, donnait un résultat peu heureux qui pouvait fausser la perception des visiteurs : ceux-ci ne comprenaient pas qu'il s'agissait d'une réparation « historique » et considéraient l'aspect maladroit du remontage comme une faute grossière de notre restauration. Cette solution avait pourtant été utilisée sur le panneau symétrique de la terrasse du Roi lépreux. Mais sur celui-ci, les reliefs en réemploi de ce panneau montrent clairement qu'il s'agit d'une reprise ancienne, à la différence des blocs du retour nord qui ne présentent pas de traces aussi évidentes de réemploi. Sur les recommandations du directeur de projet, il a donc été décidé d'enlever cette réparation tardive. Nous avons donc engagé une seconde phase de recherche de blocs pour compléter le mur d'origine. Seuls dix blocs ont pu être retrouvés et ont été remontés, puis les lacunes hautes ont été parementées de latérite en retrait. Dans ce parement ont été taillées quatre cavités circulaires dans le prolongement de celles mises au jour derrière le parement de grès correspondant aux poteaux d'une structure de bois. Ces cavités qui s'interrompent avec la tête du mur permettent d'indiquer la présence des poteaux. En tête de mur, en arrière du parement, un drain sec a été réalisé et raccordé à un regard et à une descente intégrée dans l'angle ouest du mur.

\section{Éléments mis au jour lors des travaux}

Cette anastylose a permis de mettre au jour les traces d'une structure en bois dont la base des poteaux venait s'insérer dans des logements ménagés entre la face arrière du parement et le massif de latérite. Nulle trace n'avait été trouvée sur le panneau symétrique nord ; il n'est donc pas assuré que cette structure ait pu recouvrir le passage entre les deux terrasses. Il nous semble plus probable d'envisager que ces poteaux supportaient une structure construite sur la face occidentale du perron nord où d'autres logements de poteaux ont été dégagés. Par ailleurs, ce travail a aussi montré l'existence d'une porte à battant qui fermait le passage entre les deux terrasses: la crapaudine a été retrouvée in situ et les traces d'usure que l'on y observe sont sans équivoque. Mais la compréhension d'ensemble de cette zone ne pourra être obtenue que lors de la restauration de l'escalier nord qui semble être un élément rajouté masquant en partie le dispositif original.

\section{Travaux prévus durant la seconde année}

L'anastylose entreprise a été achevée en février 1997. Les futurs travaux concerneront l'anastylose de l'escalier tardif en latérite - à la jonction orientale du retour nord avec le mur du premier état (M1) - et la réalisation d'un glacis en tête du mur qui se raccordera avec celui de la base du stupa. 


\section{Face Ouest}

\section{État avant travaux}

Dans le cadre des travaux préliminaires, un relevé d'ensemble du perron a été réalisé au $1 / 20^{\circ}$. Mais ce travail n'était guère significatif sur la face ouest du perron, car celle-ci n'ayant jamais été dégagée, elle se présentait comme un long glacis de terre d'où quelques vestiges d'alignements de latérite émergeaient entre des blocs divers. Cette situation était semblable à celle rencontrée par B. P. Groslier en 1968 sur la face ouest de la terrasse du Roi lépreux. Les dégagements y avaient alors mis au jour un long ensemble de murs redentés en latérite et un réseau de drainage. Au perron nord de la terrasse des Éléphants, il était probable de retrouver cette configuration et, au sud-ouest, l'angle des douves du palais royal.

Les moyens disponibles pour cette première année ne nous permettaient pas d'ouvrir dans le même temps un autre chantier d'anastylose en plus de ceux du mur du troisième état et du retour nord. Nous avons alors engagé le dégagement de cette face occidentale du perron en prévision de l'aménagement des abords inscrit au projet. Ces dégagements visaient tant à mettre en valeur cette face qu'à reconnaître ses raccords avec le retour nord, la terrasse supérieure du stupa et l'aile sud du perron qui la relie au perron central.

\section{Sondages}

Deux sondages préliminaires ont été réalisés sous forme de tranchées orientées est-ouest, de 3 mètres de large sur 14 de long, allant de haut en bas du glacis. TP $1 \mathrm{~W}$ devait permettre de localiser la présence du mur de latérite et TP2W offrait la possibilité d'étudier un alignement de blocs orienté est-ouest presqu'au centre du perron, qui descendait partiellement sous forme d'escalier. En fait TP1W a tout d'abord dégagé le contrefort de latérite attendu, mais nettement plus à l'ouest que prévu, révélant ainsi la présence d'un redent très proche de l'angle nordouest. TP2W a montré quant lui que les vestiges de blocs ne correspondaient que très partiellement à un gradinage de surface dont les parties ouest et sud s'interrompaient brusquement.

Ces premiers sondages nous permettaient néanmoins d'envisager une configuration comparable à celle de la face ouest de la terrasse du Roi lépreux. Nous les avons poursuivis en engageant cinq nouvelles tranchées destinées à dégager les divers panneaux du contrefort de latérite (TP3W à TP7W). Ces tranchées parallèles ont été espacées par des bermes d'un mètre de large. Au fur et à mesure du dégagement des couches, la totalité des blocs effondrés ont été numérotés et relevés en trois dimensions au tachéomètre, et la céramique collectée. Un huitième sondage a été engagé en septembre au pied de la face ouest de l'aile sud qui rejoint le perron central (cf. pl. 1, p. 216).

\section{Éléments mis au jour lors des travaux}

\section{SÉQUENCES D'OCCUPATIONS ET SUPERPOSITIONS DES STRUCTURES}

Les dégagements de la face ouest ont mis au jour une superposition de structures et de nombreux artefacts dont des éléments de couverture en plomb. L'analyse des sondages et est encore en cours mais a d'ores et déjà permit de dresser une séquence générale des occupations de cette zone inédite et de préciser quelques aspects de la métallurgie et de l'emploi du plomb à Angkor (cf.« Nouvelles données sur les couvertures en plomb à Angkor » p. 183-220). Rappelons seulement ici que la face occidentale du perron porte les traces de nombreux remaniements qui ont modifié profondément cette zone. Les aménagements les plus tardifs peuvent être rattachés à l'aménagement du stupa au sommet du perron : gradinage partiel dans le prolongement occidental de la terrasse du stupa, aménagement d'une plate-forme dallée 
construite sur le comblement de l'angle nord-est de la douve du palais royal, doublage du contrefort de latérite dans l'angle sud-est se prolongeant vers l'ouest par la base en latérite d'un alignement de poteaux, et enfin probablement réparation des parties supérieures du contrefort de latérite de l'angle nord-ouest. Sous ces aménagements ont été dégagés des structures que nous pouvons associer au règne de Jayavarman VII : un réseau de drainage relié à celui de la terrasse du Roi lépreux et un second drainage traversant la partie sud du perron, les deux se déversant dans les douves, une structure bordant la face nord de ces douves, un contrefort de latérite redenté qui limite la face occidentale du perron. Mais il est apparu que ces structures recouvraient partiellement ce que nous avons interprété comme un atelier métallurgique où une grande quantité de tuiles en plomb a été découverte. Sous ce niveau d'occupation, probablement antérieur à la fin du XIIe siècle, des vestiges du XIe siècle ont été mis au jour : la base d'une structure de bois et, bien sûr, les gradins des douves du palais royal, elles-mêmes bordées de structures légères. Enfin, un sondage a ponctuellement mis en évidence l'existence de vestiges antérieurs à cette douve. On observe donc que cette face du perron nord est aussi complexe que la face orientale, bien que toutes les séquences observées ne puissent être directement rattachées aux divers parements sculptés présents à l'est.

\section{Travaux réalisés, Moitié nord}

Dans le prolongement des dégagements et après réalisation des relevés architecturaux et archéologiques, nous avons dû envisager, en plus de la mise en valeur de cette face ouest prévue dans le projet initial, la consolidation des structures dégagées.

L'élément le plus important dans le cadre de l'aménagement de la moitié nord de cette zone est sans conteste le contrefort de latérite redenté qui maintient le remblai. Bien que ce mur ne soit pas - ou plus - parementé de grès et ne soit donc guère spectaculaire, il délimite la majeure partie de cette face occidentale, lui rend sa volumétrie d'origine - du moins à la fin du XII e siècle - et offre par ailleurs l'opportunité de rester cohérent avec le traitement de la face occidentale de la terrasse du Roi lépreux réalisé en 1968. Il permet enfin de conserver visible les états postérieurs (terrasses et gradins supérieurs au-dessus de ce mur). Par contre, les vestiges antérieurs dégagés dans la moitié nord sont situés sous le niveau du contrefort de latérite et ne se réduisent qu'à quelques blocs. Nous les avons donc ré-ensevelis.

\section{CONFORTATIONS EN SOUS-GEUVRE}

Laisser ce mur apparent imposait de le consolider afin qu'il puisse contenir la pression du remblai interne du perron. Le mur similaire de la terrasse du Roi lépreux avait été anastylosé. Reprendre ce processus présentait ici plusieurs désavantages : une durée de chantier longue, un coût important et le remontage d'un mur dont l'appareillage est assez approximatif avec des blocs à peine parallèloïdes et des joints ouverts bouchés de terre.

Nous avons donc opté pour une technique de consolidation en sous-œuvre qui évite le démontage de la structure, permet des délais et des dépenses moindres et conserve l'authenticité du parement avec ses imperfections et ses déformations. Deux problèmes essentiels étaient à résoudre : la pression du remblai et le drainage et l'évacuation des eaux. Le processus que nous avons développé pour la moitié nord - où la hauteur du mur n'est que de $3,50 \mathrm{~m}$ au maximum - consiste à réaliser une tranchée dans le remblai de terre en arrière du mur afin de dégager sa face interne jusqu'à l'assise de base, puis de couler sur une étanchéité une semelle puis une dalle de béton armé enduite sur sa face interne d'un imperméabilisant. Deux drains sont réalisés, l'un en pied et l'autre en tête de mur, et raccordés au système d'évacuation. Puis la tranchée est remblayée.

Ce processus présente néanmoins un désavantage important : celui de la sécurité. La fragilité du mur une fois la tranchée réalisée et la présence de remblai instable mis à nu ont imposé de prendre des mesures de sécurité particulières. Les travaux ont donc été exécutés après la saison des pluies, par petits tronçons avec des étaiements de sécurité et une importante 
rapidité d'exécution. La consolidation de la moitié nord a été réalisée suivant ce procédé de novembre 1996 à février 1997.

\section{PAREMENT DES LACUNES EN LATÉRITE}

De décembre à mars, un nouveau parement de latérite a été taillé et assemblé dans les lacunes hautes afin de masquer le sommet du voile de béton armé qui maintient le remblai. La hauteur exacte d'origine n'étant pas connue, puisque le sommet avait été l'objet de réaménagements importants, il n'était pas possible de restituer la volumétrie d'origine. La tête du mur a donc suivi le profil existant. Ce nouveau parement assure une cohérence de traitement tant avec les autres parties du perron qu'avec la terrasse du Roi lépreux et en particulier la face ouest traitée dans le même esprit par Groslier - à la différence près que nous avons ici dressé le nouveau parement légèrement en retrait du mur d'origine, ce qui permettra de distinguer les parties anciennes plus aisément lorsque la patine et l'oxydation des blocs neufs auront effacé toute différence de teinte. Notons cependant que, comme à la terrasse du Roi lépreux, une distinction restera toujours possible car les blocs neufs sont d'une qualité d'appareillage nettement supérieure à celle d'origine...

\section{Travaux projetés, moitié sud}

Alors que la moitié nord de la face ouest a pu être intégralement consolidée et mise en valeur durant cette première année en respectant l'enveloppe du projet, la moitié sud n'a pu être entreprise. Cette zone, au fur et à mesure de son dégagement, a montré une configuration complexe et des données nouvelles qui mettent en œuvre, pour sa restauration, des paramètres divers qui dépassent la simple mise en valeur de la face arrière du perron prévue initialement. À la fin de cette première année, il apparaît que le traitement de cette zone peut permettre non seulement la consolidation et la mise en valeur des strates historiques mises au jour, mais aussi - et surtout - de remettre en valeur un système hydraulique ancien tout en fournissant une meilleure solution pour les problèmes de drainage qui se posent sur les terrasses royales. À moins de ré-ensevelir l'ensemble des structures dégagées afin d'assurer leur conservation, nous envisageons actuellement une extension du projet d'origine. Ce nouveau projet est en discussion et le traitement de cette zone reste pour l'instant en suspens.

\section{Mur du quatrième état (M4)}

\section{État avant travaux}

Le panneau de reliefs du quatrième état orne la base orientale du stupa situé au sommet du perron nord de la terrasse des Éléphants. Il présente une composition - incomplète au sud centrée sur un Rahu, encadré par deux éléphants tricéphales dans un ensemble de danseuses et de guerriers étrangement similaires à ceux de la face orientale du mur du second état. L'extrémité septentrionale présente un angle orné de devata qui amorce un retournement vers l'ouest. Madeleine Giteau a noté que ce panneau présente certains reliefs (le Rahu et les devata) dont la facture dénote qu'ils ont été sculptés lors du retour de la monarchie à Angkor au XVIe siècle ${ }^{86}$.

Le parement de grès, conservé sur une hauteur maximum de $2 \mathrm{~m}$, s'adosse à un blocage de latérite qui sert de base au stupa. Les blocs de parement avaient été remontés avec insertion de crampons de liaison en 1934 après la chute d'un arbre. Leur base montrait par ailleurs des tassements importants qui se répercutaient dans l'ouverture de joints dans le parement, accélérant la concentration des eaux pluviales et leurs ruissellements qui altére les reliefs. Par

86. Iconographie du Cambodge post-angkorien, EFEO, Paris, 1975, p. 116-118, Pl. XIII, Ph. 13. 
ailleurs, nombre de blocs épars semblaient provenir des parties supérieures du panneau (têtes d'éléphants, danseuses...). Leur remontage avait été envisagé en 1934 mais abandonné devant l'ampleur des lacunes.

\section{Travaux réalisés}

\section{ANASTYLOSE}

Le projet présenté en 1995 prévoyait l'anastylose de ce panneau durant la seconde année. En fonction des modifications de programme et du budget alloué pour 1996, ce travail d'ampleur moyenne a pu être entrepris en mars 1997 quand l'achèvement des autres parties restaurées a permis de libérer les équipes d'ouvriers nécessaires. Après relevé détaillé au 1/20, tant de l'appareillage que des reliefs, l'ensemble du parement a été démonté. Le libage de latérite est en cours de démontage en prévision du coulage de la dalle de fondation.

\section{Éléments mis au jour lors des travaux}

\section{RECHERCHE DES BLOCS MANQUANTS}

Comme on l'a vu pour la recherche des blocs de M3, une confusion importante régnait parmi les divers éléments sculptés du perron nord, tant pour ceux remontés que pour ceux qui étaient stockés au sol. Aussi, la recherche des blocs pour M3 s'est étendue aux autres parties du perron et en particulier aux reliefs de M4 dont les éléphants tricéphales et les danseuses peuvent parfois ressembler à ceux des parties hautes de la face orientale de M3. Préalablement déposés dans des zones voisines en gardant une numérotation spécifique qui indique leur provenance, les blocs ont été classés suivant leur type de reliefs (danseuses et têtes d'éléphants, défenses, colliers, poitrails, trompes) et assemblés au sol.

Après de nombreuses tentatives, parfois infructueuses, d'importants groupes de reliefs ont finalement été constitués puis associés entre eux. Bien que nous travaillions encore à ces assemblages au sol et que les lacunes restent parfois importantes, il est désormais certain de pouvoir reconstruire le panneau de M4 avec les têtes des deux éléphants tricéphales dans toute leur hauteur, tout en garantissant l'emplacement exact de chaque bloc.

\section{SUPERPOSITIONS DES STRUCTURES}

Le démontage a été complété par un sondage à l'extrémité nord où une terrasse à l'évidence tardive recouvrait la base de M4 et la tête de M1. Ce sondage a permis de retrouver une partie du dallage d'origine de $\mathrm{M} 1$, le mur de latérite M0 qui suit en retrait d'un mètre le parement de $\mathrm{M} 1$ et un bloc sculpté provenant des éléphants de M4, dans une partie restaurée en 1954. Un second sondage ponctuel a été récemment réalisé à l'extrémité sud afin d'observer l'imbrication de la base de M4 avec une frise sculptée de hamsa. Ce sondage limité a mis au jour des fragments de feuille de bronze et quelques morceaux de nacre. Il est probable que ces objets, situés à une trentaine de centimètres sous le libage, faisaient partie d'un dépôt de fondation ${ }^{87}$.

Enfin, au moment d'achever ces lignes, alors que le remontage est déjà engagé, l'observation de la stéréotomie des blocs et les relevés complémentaires nous ont permis de confirmer la suggestion émise par M. Giteau, à savoir que M4 est constitué de panneaux de reliefs en réemploi entre lesquels quelques blocs ont été sculptés au XVIe siècle. Mieux, nous avons pu identifier avec exactitude l'emplacement d'origine des panneaux: la moitié sud provient de la lacune centrale de la face orientale du panneau de $\mathrm{M} 2$, et la moitié nord correspond à la majeure partie de celle du panneau oriental de M1 (la partie restante, au sud, a

87. Des morceaux de nacre ont déjà été dégagés dans des dépôts de fondation: au Krol Roméas des Kulen (BEFEO XXXII, p. 524) et à Vat Athvear (Rapports de la Conservation d'Angkor, septembre 1934) cf infra tableau p. 400-401. 
été réemployée au sommet de la terrasse du Roi lépreux et intégrée dans un panneau de reliefs au profil particulier, aujourd'hui reconstitué sur le perron extrême-nord). Ces réemplois soulèvent un point en apparence paradoxal : il serait logique d'envisager que le réemploi des panneaux centraux de M1 et de M2 ait été réalisé à l'occasion de l'édification de M3. Or les reliefs de cet état sont de facture nettement supérieure et de style antérieur aux quelques reliefs du XVIe siècle de M4. Il nous semble donc plus probable de considérer actuellement M3 comme antérieur à ce réemploi. Celui-ci aurait été réalisé au XVIe siècle à l'occasion d'un remodelage général de la plate-forme du perron qui aurait conduit à la mise au jour et à la récupération des parties centrales des panneaux M1 et M2. Il apparaît donc clairement que le sommet du perron nord et celui de la terrasse du Roi lépreux ont été profondément remaniés au XVIe siècle.

Notons enfin que l'identification de l'emplacement d'origine des réemplois permettrait d'envisager de les y reconstruire au lieu de les laisser au sommet du perron. Certes, ces remontages seraient particulièrement spectaculaires puisqu'ils permettraient de reconstituer la quasi intégralité des panneaux sculptés aux motifs si originaux. Mais cette solution constituerait la négation et la destruction des réaménagements du XVIe siècle au sommet du perron. Le projet de restauration visant au contraire à conserver et mettre en valeur l'épaisseur historique de l'édifice, nous avons donc maintenu la restauration de M4 comme prévue initialement. Seules des restitutions graphiques de M1 et de M2 seront réalisées...

\section{Travaux prévus durant la seconde année}

L'anastylose de ce mur ira de pair avec celle du stupa car ces deux structures se sont révélées étroitement imbriquées. Le mur de parement sera reconstruit sur sa nouvelle dalle de fondation et s'adossera sur un massif de latérite maçonnée. Des ancrages seront réalisés afin d'assurer une bonne stabilité aux têtes qui sont largement en porte-à-faux par rapport au nu du mur. Certains blocs devront par ailleurs être consolidés puisque remontés à tort dans l'éléphant de M3, ils ont été brisés par la chute de l'arbre. Enfin, deux drains recueilleront et évacueront les eaux pluviales vers le regard installé au nord-ouest du mur du retour nord.

\section{Stupa}

\section{État avant travaux}

Le stupa situé au sommet du perron nord ne présentait qu'un dallage de latérite supportant une base composée d'une assise octogonale et de trois assises circulaires de blocs de latérite dont certains ont conservé ponctuellement des traces d'enduit de chaux. Les blocs de grès supposés correspondre au sommet du stupa étaient entassés à quelques mètres à l'ouest avec de nombreux fragments divers. Outre quelques petits travaux de reprise de la base de latérite, cet édifice n'avait guère été l'objet d'attention particulière et l'on ignorait encore son profil d'origine.

\section{Dégagement et essais de remontage}

En mai 1996, les blocs qui jonchaient cette zone ont été déposés et stockés. Alors que les relevés des dallages dégagés et de la base étaient réalisés, une équipe a été chargée de reconstituer au sol le sommet du stupa. Ces premières tentatives de remontage au sol ont dû être abandonnées car l'ampleur des lacunes ne nous permettait pas de garantir l'exactitude du remontage de l'ensemble des groupes de blocs assemblés. Bien que très fragmentaires, ces assemblages au sol nous ont cependant permit de constater que ce que l'on croyait être un 
stupa «classique » en forme de cloche était en fait un motif de couronnement d'une tour - en bouton de lotus ouvert vers le haut - donc en réemploi.

Les sondages réalisés sur la face ouest ayant mis au jour plusieurs blocs pouvant provenir de ce "stupa », nous avons profité des grosses pluies qui empêchaient certains travaux de dégagement de la face ouest pour reporter deux équipes sur le stupa. Les assemblages ont été repris en les complétant avec les blocs nouvellement trouvés. Le résultat est plus satisfaisant mais reste très fragmentaire. Il permettra néanmoins de matérialiser cette forme très particulière de «stupa ». Il n'est cependant pas possible à l'heure actuelle de déterminer avec certitude la provenance de ce motif terminal, mais on note des motifs semblables tant au Bapùon qu'au Bayon. L'autre équipe a dégagé la partie ouest au pied de la terrasse du «stupa » : ces travaux n'ont montré qu'un glacis de terre comprenant de nombreux blocs et fragments sculptés jetés pêle-mêle. À une profondeur de deux mètres, soit sensiblement au niveau du départ des gradins de latérite qui recouvrent l'extension occidentale, les vestiges incomplets d'un beau dallage de grès encore en place ont été mis au jour. Les dégagements se sont arrêtés à ce niveau puisque rien, sinon la curiosité, ne justifiait de déposer ce dallage pour fouiller plus bas. Ce dallage a été l'objet d'un relevé détaillé et sera recouvert ultérieurement par un nouveau glacis de terre lors de la restauration du stupa.

\section{Travaux prévus durant la seconde année}

L'anastylose du stupa et de sa plate-forme a été engagée en avril 1997 par la dépose complète du dallage supérieur de latérite. Le socle composé d'un amas de blocs en réemploi est en cours de creusement. Une dalle sera établie et un système de drainage réalisé et raccordé au réseau d'évacuation établi à l'angle ouest du retour nord. Les éléments démontés seront ensuite reposés et scellés puis le couronnement sera reconstruit partiellement en fonction des blocs trouvés.

\section{Mur du premier état (MI)}

\section{État avant travaux}

Le mur de premier état (M1) dégagé et partiellement consolidé en 1952 présente trois tronçons aux configurations distinctes : la face orientale, les deux ailes latérales qui la bordent et deux ailes suivantes à l'ouest. Seules ces dernières n'avaient pas été recouvertes par M2.

\section{FACE ORIENTALE}

La partie centrale de la face orientale est réduite au centre à deux assises de fondations en grès et, près des angles, à quelques assises de reliefs sculptés indiquant la présence de chevaux heptacéphales. Les reliefs manquants ont été identifiés dans la moitié nord du panneau de M4 et dans un panneau de reliefs originellement situé au sommet de la terrasse du Roi lépreux et actuellement reconstitué sur son perron extrême-nord. En arrière des vestiges de M1 file un mur de latérite dont le fruit constaté dès son dégagement avait motivé la pose d'un contrefort de béton armé. Les parties supérieures de ce mur avaient été complétées par une maçonnerie de latérite. Dans sa moitié nord, un escalier permettant d'accéder depuis la plate-forme du perron avait été réalisé suivant le même procédé dans l'espace laissé entre Ml et le mur de latérite, alors que l'espace qui lui fait face au sud a été comblé d'un blocage de latérite.

\section{FACES LATÉRALES ORIENTALES}

Les faces latérales nord et sud de l'extrémité orientale présentent de beaux reliefs de chasse à l'éléphant. Partiellement démontés et consolidés en 1952 par des crampons qui les relient au mur de latérite qui file en arrière, ces reliefs semblent présenter une bonne stabilité : 
les rejointoiements et les lacunes comblées de latérite enduite au ciment ne montrent pas d'indice laissant supposer un déversement de ces panneaux sous l'éventuelle pression du remblai interne de la terrasse. L'efficacité de la restauration antérieure peut par ailleurs s'expliquer par la faible hauteur des panneaux à cet endroit et par la profondeur du mur arrière de latérite qui maintient le remblai. Une partie de la face nord présente cependant un léger dévers.

\section{FACES LATÉRALES OCCIDENTALES}

Les faces latérales nord et sud de la partie occidentale, couvertes elles aussi de reliefs de chasse à l'éléphant, ont subi diverses reprises partielles à la suite des risques d'effondrement qui y furent constatés (1917 et 1954). Leurs parties supérieures présentent de nombreux blocs qui ne sont, à l'évidence, pas à leur place. L'état des lieux nous laisse penser que ces panneaux, plus élevés que les précédents, sont plus sensibles à la pression du remblai interne (le panneau sud montre d'ailleurs un dévers évident).

Quant aux panneaux nord et sud, celui du sud ne présente plus - après une réparation antérieure au XXe siècle, un effondrement en 1912, un remontage en 1916 et une reprise en 1917 - que quelques assises d'origine à la base d'un remontage hétéroclite et sans aucun soucis ni de l'appareillage original, ni de la cohérence des reliefs. Le panneau symétrique au nord est dans un état plus satisfaisant bien que ces parties supérieures montrent aussi des remontages peu heureux dont le dernier date de 1954 pour l'aménagement des échiffres de l'escalier nord. Cette restauration restitue par ailleurs un niveau erroné du perron.

\section{Sondages}

Le projet de restauration prévoyait durant la première année l'anastylose de l'intégralité des panneaux de M1. Aussi, nous avons réalisé les relevés graphiques nécessaires et avons engagé quelques sondages préliminaires afin de reconnaître la configuration exacte des structures à anastyloser. Ces sondages ayant montré une stabilité suffisante pour les panneaux nord et sud de l'extension orientale, nous avons repoussé la restauration des autres panneaux de ce mur du premier état (panneau central et panneaux occidentaux) afin d'avoir les moyens suffisants pour entreprendre l'anastylose du mur du troisième état (M3). Le mur du premier état n'a donc fait l'objet cette année que de dégagements et de sondages. Auparavant, les blocs empilés sur la plate-forme du perron depuis 1953 ont été enlevés, sans numérotation particulière puisque leur provenance était indéterminée, et ont rejoint le champ de dépose. Ils ont par la suite fait l'objet des recherches engagées pour reconstituer les lacunes de M3 et de M4.

PANNEAU CENTRAL

Un premier sondage, implanté au centre du panneau oriental a mis au jour la constitution des assises de fondation: sous l'assise correspondant au niveau du dallage extérieur de M1, une assise de réglage en grès sur une assise de latérite repose sur un remblai de sable pur.

FACES LATÉRALES ORIENTALES

Une partie de la face latérale nord présentant un léger dévers, nous avons réalisé un second sondage pour observer la configuration à cet endroit. Après dépose du pavement préalablement numéroté, le sondage a été poursuivi en profondeur jusqu'au niveau de l'assise de base de M1. Le sondage a permis de réaliser une coupe du mur et s'est heurté, à une profondeur de $3,73 \mathrm{~m}$ à un sol composé de bloc de latérite dont la présence reste obscure. Le sondage indique une stabilité satisfaisante face aux pressions du remblai peu élevé $(3,15 \mathrm{~m})$ et ne permet guère de justifier à l'heure actuelle une anastylose intégrale. 
FACES LATÉRALES OCCIDENTALES

La restauration des faces latérales nord et sud de la partie occidentale, dont le démarrage était prévu durant le second semestre, n'a pu être entreprise avec les crédits alloués cette année. Seul un sondage réalisé récemment à l'occasion du démontage du panneau de reliefs situé sur le perron (M4) a permis d'observer le massif interne en arrière de l'angle nord. Ce sondage a mis au jour la tête d'un mur de latérite séparé du parement d'environ 1 mètre, qui semble être la continuité du mur de latérite M0. Enfin, ce sondage a souligné la nécessité d'incorporer en tête de mur un drainage adéquat.

\section{Éléments mis au jour lors des travaux}

À l'occasion du sondage réalisé au centre du panneau oriental, nous avons dégagé la base d'une consolidation de 1953 constituée d'un blocage de latérite refermant l'espace entre le parement de grès de $\mathrm{M} 1$ et le mur de latérite en arrière. Nous avons constaté tout d'abord que ce blocage ne descendait pas jusqu'au niveau nécessaire et laissait apparaître à la base le remblai composé d'éclats de grès, de fragments de latérite et de terre. En rafraîchissant légèrement la coupe de ce remblai, nous avons eu l'heureuse surprise d'y mettre au jour une très belle jarre à glaçure noire quasi complète. La localisation de cette jarre et la configuration dans laquelle nous l'avons dégagée - à savoir brisée en deux moitiés au niveau des hanches, et ses deux moitiés disposées horizontalement l'une à côté de l'autre, mais tête-bêche semblent indiquer que cette poterie constitue un dépôt rituel.

On ne peut donc que s'interroger sur la présence d'un dépôt à cet endroit, entre le parement de grès et le mur de latérite. Cette configuration, présentant un mur de parement de grès séparé de son contrefort de latérite par plus d'un mètre de pierraille provenant de taille, est exceptionnelle et ne se retrouve, à notre connaissance, que dans d'autres tronçons des terrasses royales. Il nous semble à l'heure actuelle que ces deux murs pourraient ne pas être contemporains. H. Marchal avait d'ailleurs envisagé cette hypothèse lors de ses dégagements de 1952. Il l'abandonna faute, croyons-nous d'éléments probants, mais on sait depuis les travaux de B.-P. Groslier, que les terrasses royales recouvrent un état antérieur en latérite. Il reste difficile de savoir exactement où cet auteur a observé ces vestiges, mais nous croyons que le mur de latérite en arrière du parement de grès (M1) pourrait correspondre à ce premier état - que nous nommons MO. La présence d'un dépôt entre ces deux états semble confirmer cette hypothèse. Le dépôt dégagé serait donc contemporain de l'édification de M1.

\section{Travaux prévus durant la seconde année}

En fonction du budget alloué pour la seconde année, les travaux d'anastylose pourront être entrepris sur les faces latérales occidentales et sur le panneau de latérite (M0) de la face orientale. Les premiers s'accompagneront de recherche de blocs afin de combler les lacunes et de corriger les nombreuses « réparations » antérieures. Les lacunes restantes en parties hautes recevront un parement de latérite en retrait suivant le procédé utilisé par ailleurs. Sur la face orientale, les observations permettent actuellement d'envisager une consolidation en sousœuvre comparable à celle réalisée cette année sur le contrefort de la moitié nord de la face ouest. Des démontages partiels seront néanmoins probablement nécessaires pour corriger certains dévers importants. 
Mur du deuxième état (M2)

\section{État avant travaux}

Le mur de reliefs du second état (M2) n'est plus soumis aux pressions du remblai interne depuis les dégagements de 1953. Il se présente donc désormais comme un simple mur haut de $3,50 \mathrm{~m}$. La qualité de conservation exceptionnelle des reliefs de sa face orientale, préservés pendant des siècles dans le remblai de $\mathrm{M} 3$, contraste fortement avec celle des reliefs des faces latérales nord et sud, où la surface du grès montre de nombreux types d'érosion. On observe sur chaque face des fissures qui cisaillent les panneaux et trahissent d'importants tassement des angles sud-est et nord-est.

Les panneaux ont conservé leur aplomb hormis celui du nord qui montre en sa moitié orientale un dévers vers l'extérieur. Ce problème semble avoir existé depuis longtemps puisque c'est ce qui avait motivé la dépose en 1952 de la moitié supérieure du tronçon concerné, et sa repose avec une verticalité plus satisfaisante, mais sans traiter ni la moitié inférieure qui conserve son dévers, ni les fondations. Il résulte de cette restauration un joint ouvert rejointoyé à la jonction avec les assises non déposées.

\section{Travaux réalisés}

\section{DÉGAGEMENT ET ÉTUDE DES FONDATIONS}

Le projet déposé en 1995 s'attachait à remédier aux tassements des angles et prévoyait pour ce mur une reprise en sous-œuvre sans démontage sur des dalles préfabriquées en béton armé enduites d'imperméabilisant. Suivant ce projet, les bases intérieures et extérieures du mur ont été dégagées en juillet 1996 afin de compléter les relevés avant travaux et de préciser la conception des dalles de reprise en sous-œuvre. Ces dégagements ont révélé une configuration des fondations remarquablement importante puisque le parement repose sur une large semelle composée de deux assises de grès et de latérite. Par ailleurs, la face interne du mur présente encore un dallage de grès (composé de blocs en réemploi) qui longe la base de M1. Les confortations prévues se sont donc révélées inadéquates dans la configuration rencontrée, puisque contrairement aux fondations observées sur d'autres structures du même type (terrasse du Roi lépreux ou M1 par exemple), celle de M2 est d'une part nettement plus profonde, et d'autre part, composée de - au moins - deux rangées de blocs avec joints non traversants. Cette semelle apparaissait alors assez stable pour supporter les panneaux de reliefs sur lesquels ne s'exerce plus de poussée latérale. Pour vérifier la stabilité de $M 2$, en particulier durant les travaux d'anastylose de $M 3$, une série de témoins micrométriques ont été posés sur les fissures liées à des tassements différentiels. Après un an d'observation, ces témoins n'ont pas montré de mouvement tendant à indiquer l'accentuation des tassements, et ce malgré l'ouverture de fouilles de dégagement au pied des murs jusqu'à l'assise de base, en prévision de l'insertion d'un drainage périphérique. Ce dernier demeure cependant nécessaire pour éviter les stagnations d'eaux pluviales et diminuer les remontées capillaires.

\section{DÉMONTAGE PRÉVENTIF}

Par prudence, les éléphants d'angles orientaux de M2 ont cependant été démontés afin d'éviter d'éventuels effondrements lors des travaux d'anastylose de M3. De même, le naga qui surplombait le panneau sud de M2 fut déposé. Tous ces blocs ont reçu une numérotation et sont entreposés dans des zones distinctes sur le champ de dépose oriental.

\section{IDENTIFICATION DE BLOCS D'ORIGINE}

À l'occasion des sondages de la face occidentale du perron ont été mis au jour deux blocs sculptés provenant du sommet du panneau oriental de M2. Un troisième a récemment été 
identifié lors de nos recherches des blocs lacunaires de M3 et de M4 parmi les blocs qui étaient auparavant stockés sur la plate-forme devant M4. Ces trois blocs ont donc été remontés à leur place d'origine. Les blocs de l'importante lacune centrale qui éventre la face orientale ont été identifiés : ils ont été réemployés au XVIe siècle pour constituer la moitié sud de M4 (cf. supra p. 391).

Dans les panneaux de reliefs de la face est de M2, deux petites lacunes ponctuelles, dont l'une avait été comblée en 1953 d'un bloc de grès en réemploi dont les dimensions ne convenaient qu'approximativement, ont été bouchées par insertion d'un nouveau bloc de grès taillé aux dimensions voulues. Leur surface sera taillée pour s'accorder aux volumes des reliefs voisins.

\section{Travaux prévus durant la seconde année}

Suivant les disponibilités budgétaires, les travaux prévus pour la seconde année de chantier concerneront principalement l'installation en pied des panneaux de M2 d'un double réseau de drainage (intérieur et extérieur) qui sera rattaché au réseau général du perron nord.

Nous envisageons actuellement une intervention ponctuelle de consolidation et de reprise de l'appareillage supérieur dans l'angle nord-est avant le remontage de son éléphant d'angle. Nous avons vu plus haut que cette zone avait déjà été en partie démontée en 1952. Mais les rejointoiements faits à cette époque montrent que la partie supérieure a continué depuis à se déverser vers l'extérieur. Par contre, nul mouvement n'est visible dans les parties inférieures. L'intervention consistera donc en la dépose minimale des blocs déjà démontés en 1952, à l'enlèvement des rejointoiements, à la consolidation de la semelle de base puis au remontage avec insertion de crampons en inox pour les blocs en porte-à-faux de l'éléphant.

\section{Budget et délais}

Les travaux réalisés durant cette première année d'activité ne suivent que partiellement le planning prévu dans le projet, mais ont permis de mener à bien certaines des tâches les plus importantes.

Le chantier s'est adapté en premier lieu au budget alloué : le volume de travaux a donc été réduit et n'a pu concerner que les anastyloses du mur de retour nord et de M3. La première est achevée, la seconde en cours de finition. Dans leur continuité ont été entreprises celles du stupa et de M4 auxquelles succédera celle des parties occidentales de M1.

En parallèle aux travaux d'anastylose, les sondages préliminaires ont mis au jour de nombreuses données nouvelles qui se répercutent tant sur la restauration projetée que sur la connaissance historique du monument, l'étroite liaison de ces deux domaines étant l'un des principes guidant notre intervention. Ces nouvelles informations ont permis de revoir et de réduire les consolidations prévues pour M2 et partiellement pour la partie orientale de M1.

À l'inverse, la face ouest a révélé des structures complexes d'une ampleur importante et, donc, de nouveaux besoins de restauration. La moitié nord de cette zone a pu faire l'objet cette année d'une consolidation en sous-œuvre «économique » et sa mise en valeur est désormais achevée. Par contre, la restauration et l'aménagement de la moitié sud mettent en œuvre des paramètres divers qui, vu le budget alloué, posent encore des problèmes dont les répercussions seront fondamentales pour l'une des interventions principales de nos travaux, à savoir la mise en place d'un système de drainage.

Malgré ces données nouvelles, on doit souligner que la durée des travaux de restauration du perron nord de la terrasse des Éléphants reste principalement liée aux impératifs budgétaires et à l'enveloppe globale du projet. Le rythme prévu n'ayant pu être suivi cette 
année avec le budget alloué, il est évident que le délai de deux ans envisagé en 1995 ne pourra être tenu.

\section{Éléments mis au jour lors des travaux}

Alors que le nombre de fouilles et de travaux réalisés sur le perron depuis le début du siècle ne nous permettait pas d'envisager de mettre au jour beaucoup d'éléments nouveaux, les travaux réalisés cette année se sont révélés riches en renseignements :

Les anastyloses et divers sondages ont permis de clarifier l'imbrication des divers états en dissociant par exemple M1 de M0, ou en identifiant les réemplois de M4 comme provenant de M1 et M2 - et d'obtenir une meilleure connaissance structurelle des parties de l'édifice.

En parallèle, les recherches des blocs lacunaires de M3 et de M4 ont identifié de nombreux éléments sculptés inédits qui seront reconstruits et rendront ainsi une nouvelle monumentalité à ces panneaux du perron.

L'anastylose de M3, dont une nouvelle datation est proposée, a mis au jour un réseau de dépôts de fondation dont les éléments apportent des données inédites pour l'étude des rituels religieux de fondation. Outre un alignement de tortues en plomb, ont été dégagés des «embryons » contenant des matériaux végétaux exceptionnellement bien conservés.

Enfin, les dégagements de la face occidentale ont révélé une superposition d'aménagements qui complète celle de la face orientale. Lors des travaux ont été découvertes de nombreuses tuiles en plomb qui suggèrent l'existence d'un atelier métallurgique à cet endroit. Ces tuiles en plomb inédites, dont l'existence n'était que suggérée par une brève description de Zhou Daguan en 1295, offrent une nouvelle vision de la couverture particulière de certaines architectures palatiales en bois.

Notons cependant que les financements du projet ne permettent pas d'effectuer les analyses des divers artefacts découverts (dépôts, pièces et scories métalliques...) afin de pousser plus loin leur étude.

Christophe POTTIER 


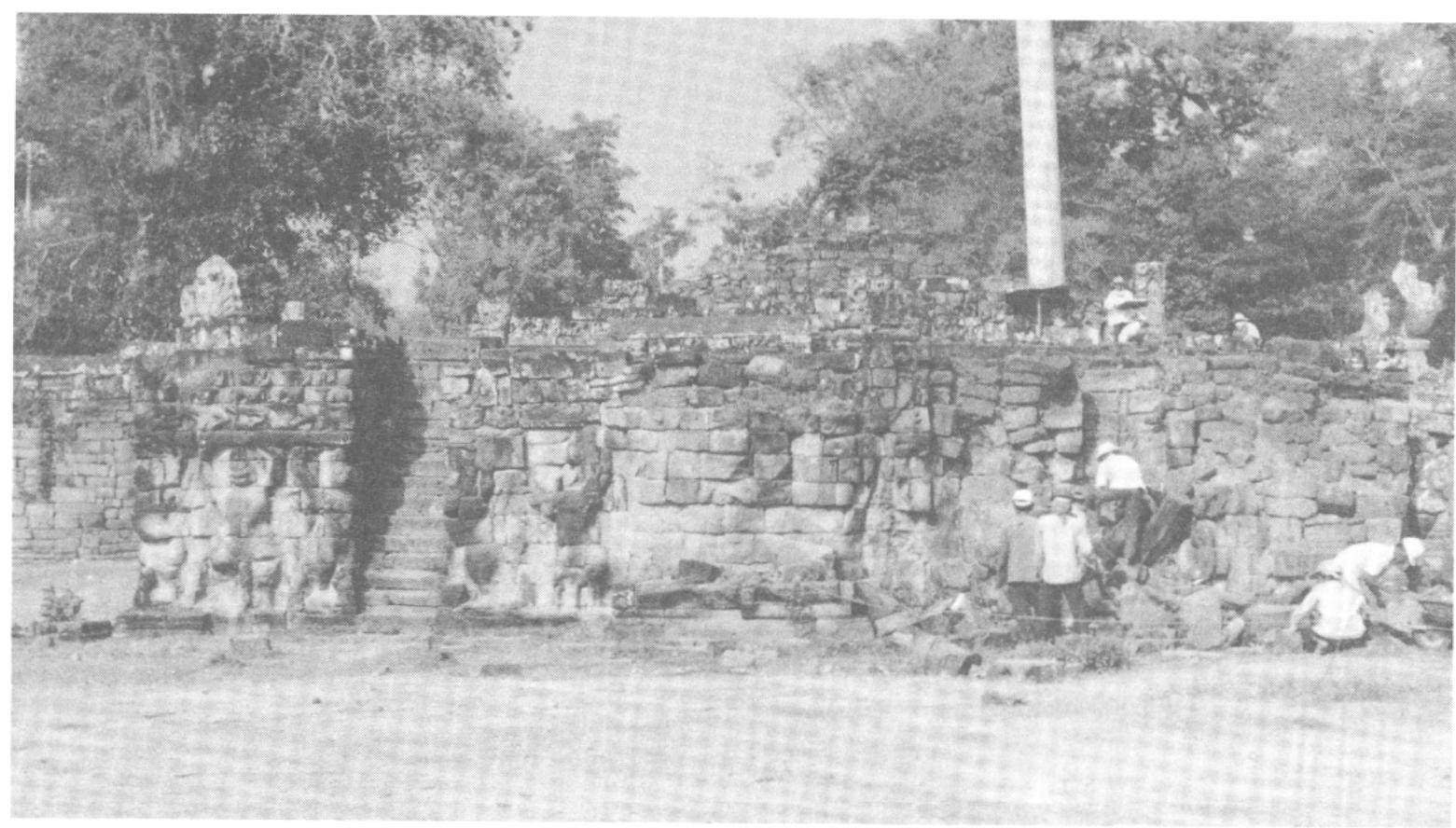

Photo 1 : Face orientale du perron nord avant dégagement, mars 1996.

Photo 2 : Remontage de M3, atril 1997.

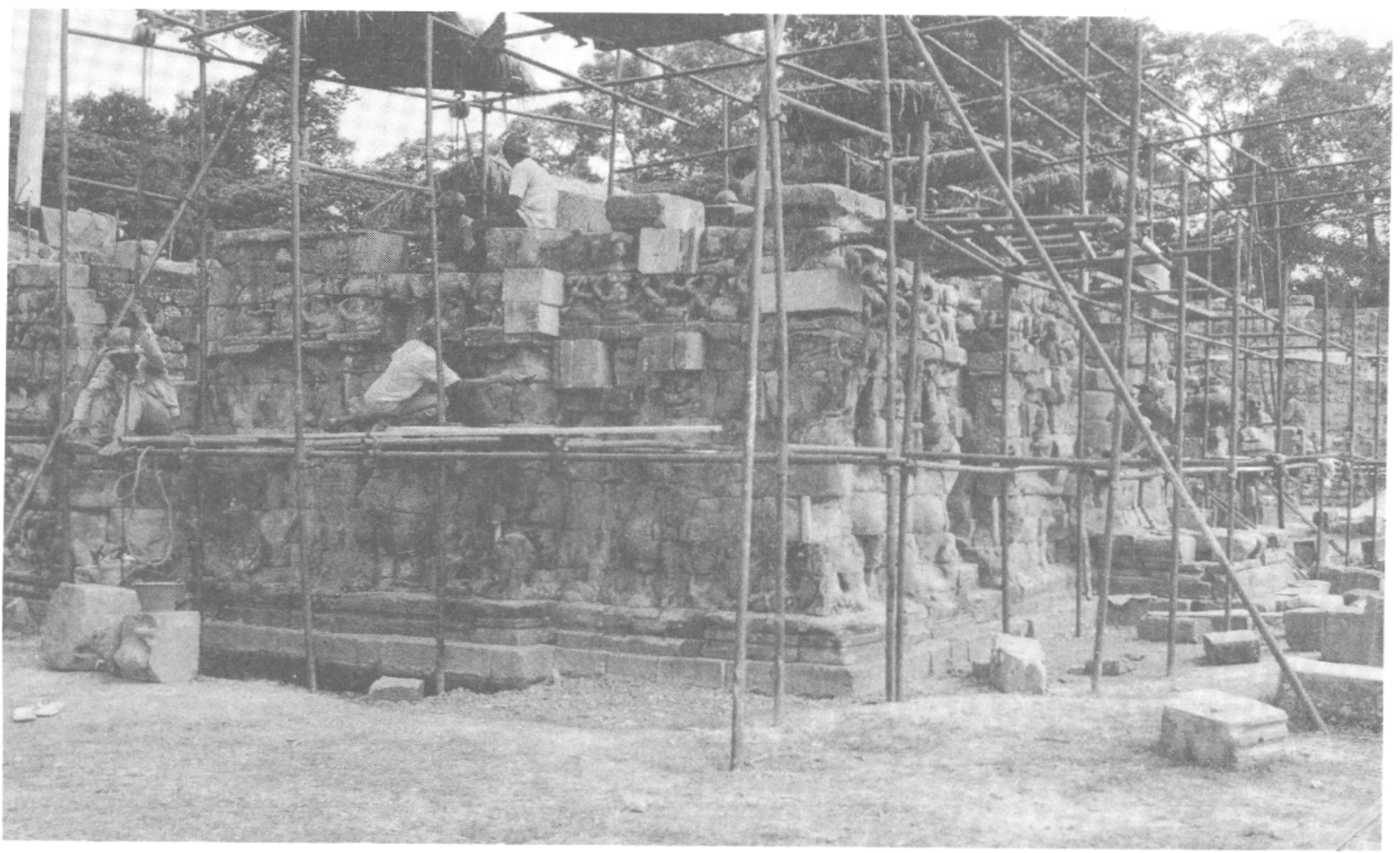




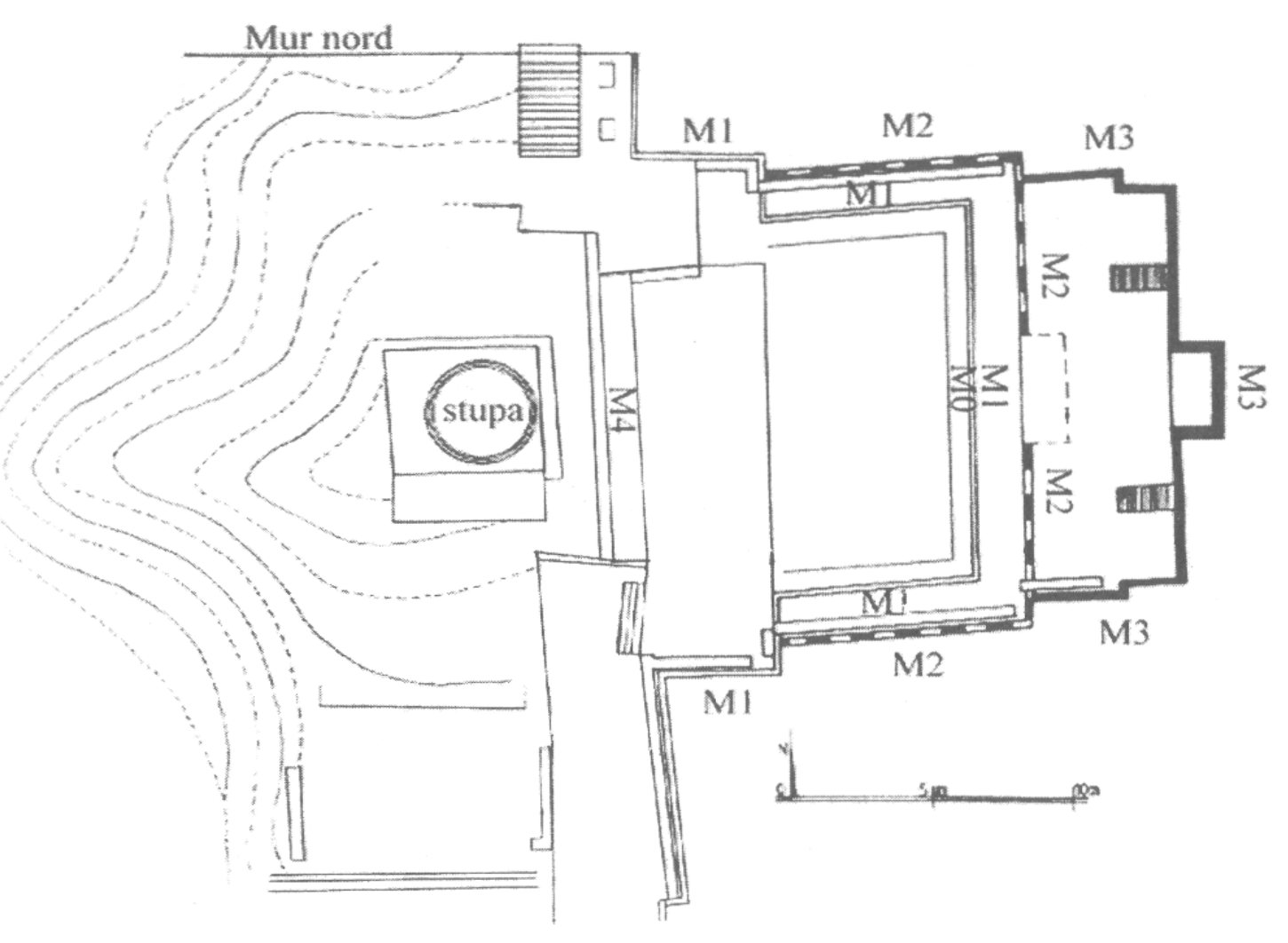

Fig. 1: Plan d'ensemble du perron nord de la Terrasse des éléphants avant travaux.

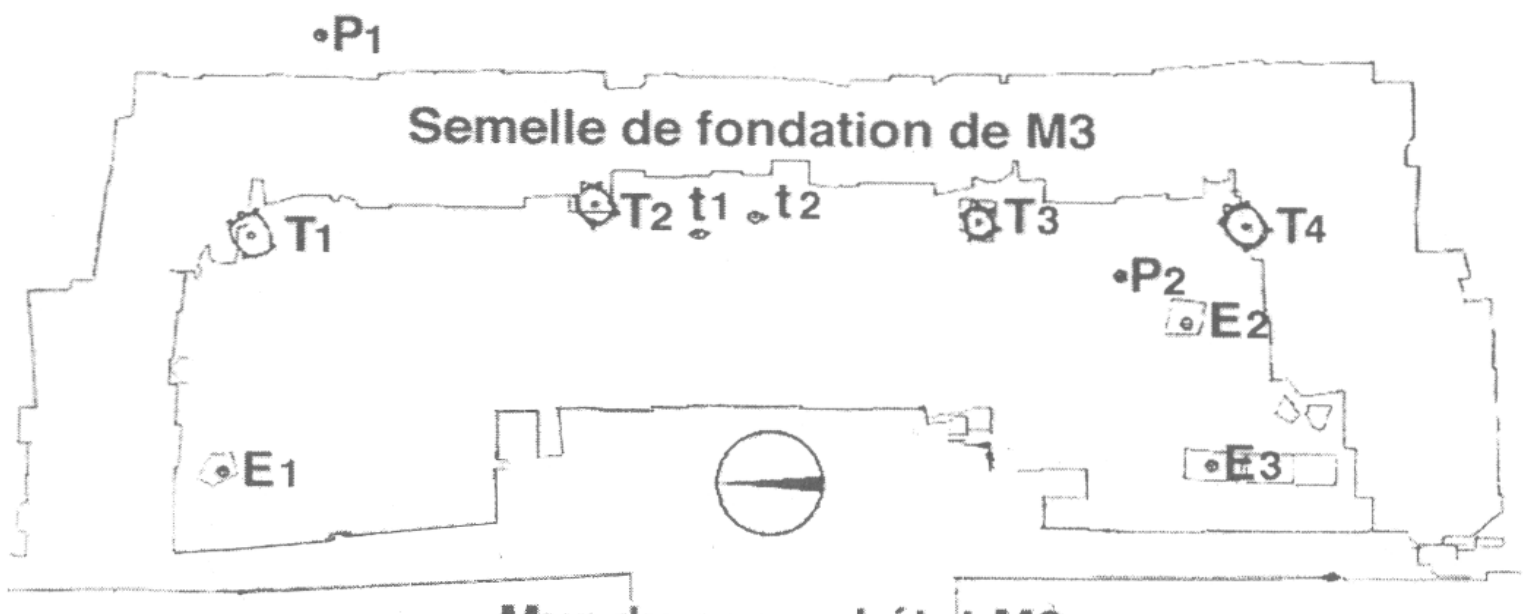

Mur du second état M2

Fig. 2: Fondation de M3, localisation des dépôts de fondation. 


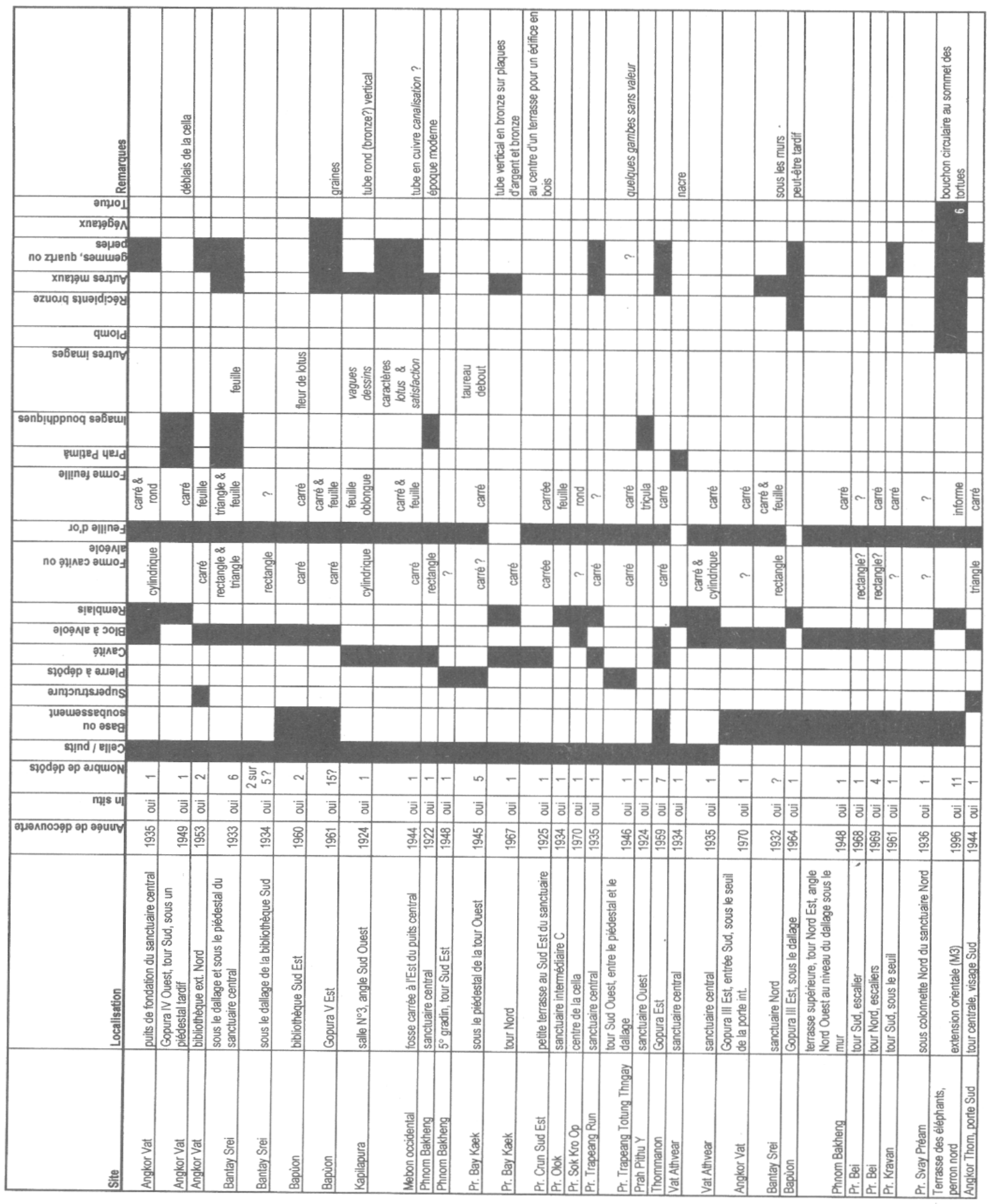

Tableau des dépôts de fondation mis au jour par la conservation d'Angkor.

(1/2) 


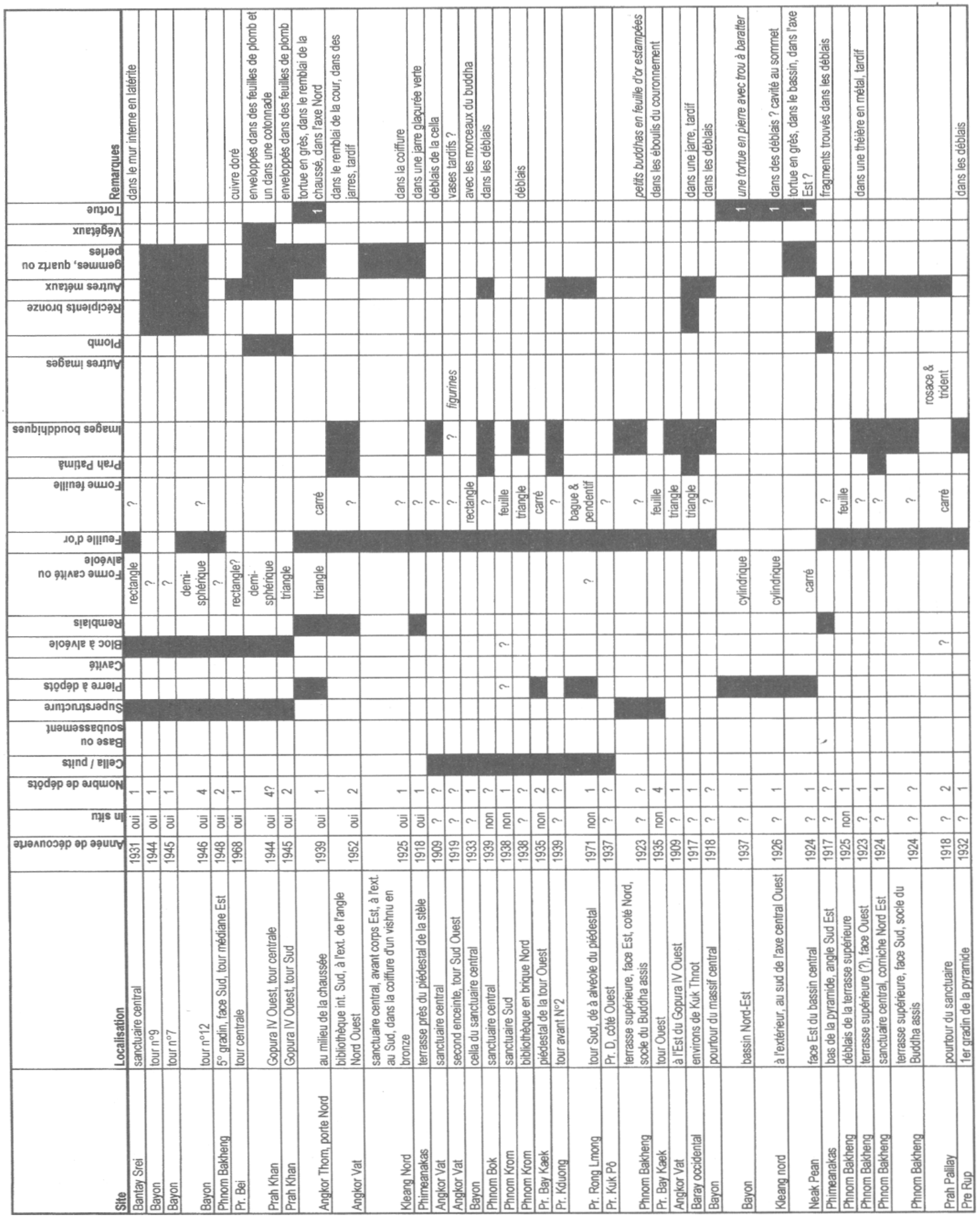

Tableau des dépôts de fondation mis au jour par la conservation d'Angkor.

(2/2) 